\title{
BMJ Open Prospective meta-analysis protocol on randomised trials of renin-angiotensin system inhibitors in patients with COVID-19: an initiative of the International Society of Hypertension
}

\begin{abstract}
Sonali Rukshana Gnanenthiran (1) , ${ }^{1}$ Claudio Borghi, ${ }^{2}$ Dylan Burger, ${ }^{3}$ Fadi Charchar, ${ }^{4}$ Neil R Poulter, ${ }^{5}$ Markus P Schlaich, ${ }^{6}$ Ulrike Muscha Steckelings, ${ }^{7}$ George Stergiou, ${ }^{8}$ Maciej Tomaszewski, ${ }^{9}$ Thomas Unger, ${ }^{10}$ Richard D Wainford, ${ }^{11}$ Bryan Williams (D) , ${ }^{12}$ Anthony Rodgers, ${ }^{1}$ Aletta E Schutte (D) ${ }^{1}$
\end{abstract}

To cite: Gnanenthiran SR, Borghi C, Burger D, et al. Prospective meta-analysis protocol on randomised trials of renin-angiotensin system inhibitors in patients with COVID-19: an initiative of the International Society of Hypertension. BMJ Open 2021;11:e043625. doi:10.1136/ bmjopen-2020-043625

- Prepublication history and additional material for this paper is available online. To view these files, please visit the journal online (http://dx.doi.org/10. 1136/bmjopen-2020-043625).

Received 09 August 2020 Revised 24 December 2020 Accepted 26 January 2021

Check for updates

(C) Author(s) (or their employer(s)) 2021. Re-use permitted under CC BY-NC. No commercial re-use. See rights and permissions. Published by BMJ.

For numbered affiliations see end of article.

Correspondence to Professor Aletta E Schutte; a.schutte@unsw.edu.au

\section{ABSTRACT}

Introduction Whether ACE inhibitors (ACEi) or angiotensin II receptor blocker (ARB) therapy should be continued, initiated or ceased in patients with COVID-19 is uncertain. Given the widespread use of ACEi/ARBs worldwide, guidance on the use of these drugs is urgently needed. This prospective meta-analysis aims to pool data from randomised controlled trials (RCTs) to assess the safety and efficacy of ACEi/ARB therapy in adults infected with SARS-CoV-2.

Methods and analysis RCTs will be eligible if they compare patients with COVID-19 randomised to ACEi/ ARB continuation or commencement versuss no ACEi/ARB therapy; study duration $\geq 14$ days; recruitment completed between March 2020 and May 2021. The primary outcome will be all-cause mortality at $\leq 30$ days. Secondary outcomes will include mechanical ventilation, admission to intensive care or cardiovascular events at short-term follow-up ( $\leq 30$ days) and all-cause mortality at longerterm follow-up (>1 month). Prespecified subgroup analyses will assess the effect of sex; age; comorbidities; smoking status; ethnicity; country of origin on all-cause mortality. A search of ClinicalTrials.gov has been performed, which will be followed by a formal search of trial registers, preprint servers, MEDLINE, EMBASE and Cochrane Central Register of Controlled Trials to identify RCTs that meet inclusion criteria. To date, a search of ClinicalTrials.gov identified 21 potentially eligible trials for this meta-analysis. We will request trial investigators/sponsors to contribute standardised grouped tabular outcome data.

Ethics and dissemination Ethics approval and informed consent will be the responsibility of the individual RCTs. Dissemination of results will occur by peer-reviewed publication. The results of our analysis can inform public health policy and clinical decision making regarding ACEi/ ARB use in patients with COVID-19 on a global scale.

\section{INTRODUCTION}

Renin-angiotensin system (RAS) inhibitors, including ACE inhibitors (ACEi) and

\section{Strengths and limitations of this study}

- First prospective meta-analysis of randomised controlled trials assessing the safety and efficacy of ACE inhibitors (ACEi)/angiotensin II receptor blocker (ARBs) in adults with COVID-19.

- This meta-analysis uses a collaborative international approach to allow pooling and dissemination of results. This has the potential to inform international public health policy and clinical decision making for ongoing ACEi/ARB use in patients with COVID-19.

- Randomised controlled trials are currently under way with some having the potential to be underpowered. Pooling of data will overcome this shortcoming.

- The completion of these trials prior to data pooling is a limitation, as is the willingness of trialists to collaborate in data sharing.

angiotensin II receptor blockers (ARBs), are the most widely prescribed antihypertensive treatments globally, used by hundreds of millions of people worldwide. ${ }^{1}$ ACEi/ARB therapy are not only first-line agents for the treatment of hypertension, but are also the cornerstones of treating cardiovascular and kidney disease such as heart failure, coronary heart disease, diabetes and chronic kidney disease (CKD). However, infection with SARS-CoV-2 involves the viral spike protein attaching to the ACE2 receptor to infect epithelial cells in the respiratory tract, ${ }^{2}$ with increased binding affinity a key determinant of pathogenicity. ${ }^{3}$ Animal studies have demonstrated that ACEi/ARB therapy may upregulate ACE2 receptor expression ${ }^{45}$ and produce increased cardiac ACE2 mRNA levels, which may promote viral cell invasion. ${ }^{46}$ Upregulated expression of ACE2 receptors on the 
cell surface has been postulated to increase the risk of infection with SARS-CoV-2 and the disease severity, with subsequent life-threatening complications. ${ }^{78}$ At the same time, data from animal studies suggest that increased ACE2 expression secondary to ACEi/ARB use might have protective benefits on cardiac, kidney and pulmonary function and thus reduce the severity of COVID- $19 .{ }^{9}$

Observational retrospective studies in humans and meta-analyses of these studies suggest that there is no adverse effect of RAS blockade on COVID-19 severity and outcome ${ }^{10-16}$ but there may be possible protective benefits including reduced rates of mortality, ${ }^{17}$ critical disease $^{15}$ and admission to intensive care.$^{18}$ Observational studies, even rigorous ones, can still have multiple sources of bias, and thus, more robust evidence is needed for sound clinical decision making. Randomised controlled trials (RCTs) are needed to mitigate this risk. However, to date, reliable data from RCTs are unavailable to guide clinical decision-making. As a result, it is uncertain whether ACEi/ARB therapy should be continued, withdrawn or initiated in patients with COVID-19.

International hypertension, cardiovascular and nephrology societies have consistently recommended that patients continue ACEi/ARB therapy during the COVID-19 pandemic, on the basis of the strong and welldocumented evidence on their protective effects, but identify a need for more reliable human data. ${ }^{19-23}$ There are multiple RCTs in process, which will better inform clinical decision making rather than relying on observational human studies ${ }^{10-14}$ and inconsistent animal data. ${ }^{46}$ Most of the RCTs under way are small to moderate in size ( $40 \%$ aim to recruit less than 250 participants), with many unlikely to meet their recruitment targets. These trials are also unlikely to be powered to answer questions regarding subgroup populations, including whether there is utility of ACEi/ARB therapy in patients with COVID-19 with concomitant hypertension, cardiovascular or kidney disease. Given the uncertainty of ACEi/ ARB use in those with COVID-19, some trials are starting ACEi/ARB therapy for possible benefit, while other trials are stopping the same therapy due to concerns about harm. These RCTs are not completely free from bias, but nevertheless represent a higher quality of evidence than observational studies. A prospective meta-analysis led by the International Society of Hypertension will, therefore, be an ideal approach to address these limitations, as well as promoting international collaboration. This approach entails studies to be identified, evaluated and determined to be eligible before the results of any included studies are known or published, thereby avoiding some of the potential biases inherent in standard, retrospective meta-analyses.

\section{Objectives}

We will, therefore, perform a prospective meta-analysis of RCTs recruiting patients with COVID-19 to assess the safety and efficacy of ACEi/ARB therapy compared with those not on ACEi/ARBs. As primary outcome, pooled data will be used to assess all-cause mortality associated with ACEi/ARB therapy compared with those not on ACEi/ARBs stratified by age, comorbidity, sex, ethnicity and trial characteristics.

\section{METHODS AND ANALYSIS Protocol design}

For this meta-analysis, we will only include RCTs to minimise the impact of bias and confounding. We herewith describe our methods as per the Preferred Reporting Items for Systematic Reviews and Meta-Analyses for protocol (PRISMA) recommendations. ${ }^{24}$ Final reporting of this study will be compliant with the main PRISMA statement.

\section{Eligibility criteria}

For clinical trials to be eligible for inclusion, the following criteria must be met: (1) RCTs recruiting between March 2020 and March 2021; (2) aged $\geq 18$ years; (3) laboratory confirmed SARS-CoV-2 infection; (4) comparison of patients randomised to ACEi/ARB versus no ACEi/ARB therapy; (5) findings reported in English; (6) trial duration $\geq 14$ days and (7) oral administration of ACEi/ARB therapies.

We will include trials that investigate continuation vs cessation of ACEi/ARB among patients currently treated with ACEi/ARB; and trials that report initiation of ACEi/ $\mathrm{ARB}$ vs control in those not currently treated with such therapies. These will be pooled in an ACEi/ARB vs no ACEi/ARB meta-analysis, with appropriate prespecification of preplanned sensitivity analyses from the two groups of trials given the fundamental differences. Studies must contain sufficient detail and be able to provide at least one outcome outlined within our data extraction form (table 1) and be reported using an intention to treat basis. If an RCT consists of multiple arms, we will include only the relevant arms. The exclusion criteria will be at the discretion of each individual trial.

The intervention will comprise continuation or initiation of ACEi/ARB therapy. The control will be discontinuation of current ACEi/ARB therapy, substitution with an equivalent dose of non-ACEi/ARB therapy, placebo or usual care.

\section{Outcomes}

The primary outcome will be all-cause mortality at $\leq 30$ days. Secondary outcomes will include mechanical ventilation at $\leq 30$ days; admission to intensive care at $\leq 30$ days; myocardial infarction at $\leq 30$ days, revascularisation at $\leq 30$ days, congestive cardiac failure at $\leq 30$ days; pulmonary embolism and/or deep vein thrombosis at $\leq 30$ days, hospitalisation at $\leq 30$ days and acute kidney injury (defined as per each individual RCT) at $\leq 30$ days; all-cause mortality at $>1$-month follow-up. Standardised grouped tabular deidentified data will be requested from triallists for both short-term ( $\leq 30$ days) and longer-term 
Table 1 Outcome grouped tabular data

No ACEi/ARB ACEi/ARB

\begin{tabular}{l}
\hline Baseline characteristics \\
Mean age \pm SD (years) \\
Sex, $n$ \\
Men \\
Women \\
Medical history, $n$ \\
Hypertension \\
Diabetes \\
Chronic kidney disease \\
Cardiovascular disease \\
Chronic obstructive \\
pulmonary disease \\
Smoking \\
Ever-smoked \\
Non-smoker \\
Outcomes for $\leq 30$ days \\
Total no of patients in the \\
group, $n$
\end{tabular}

All-cause mortality

Myocardial Infarction

Congestive cardiac failure

Revascularisation

Admission to intensive care

unit

Need for mechanical
ventilation

\section{Hospitalisation (if outpatient}

study)

\section{Pulmonary embolism/deep}

vein thrombosis

Acute kidney injury

Outcomes > 1 month, $n$

All-cause mortality

${ }^{*} \mathrm{~N}=$ number, $\mathrm{SD}, \mathrm{ACEi}, \mathrm{ARB}$; acute kidney injury: decline in kidney function or urine output from baseline.

ACEi, ACE inhibitors; ARB, angiotensin II receptor blocker; eGFR, estimated glomerular filtration rate; $\mathrm{MeSH}$, medical subject headings.

(>1 month) follow-up where available (table 1). Individual identifiable patient data will not be requested.

\section{Search strategy and searching sources}

An electronic search of ClinicalTrial.gov was performed to identify potential ongoing trials for inclusion in the meta-analysis. Figure 1 summarises the trial search as of August 2020 performed using the search terms: coronavirus 2019, COVID-19, SARS-CoV-2, RCT, ACEi and/or ARB. A total of 21 trials were identified which potentially met the eligibility criteria (figure 1 , table 2 ). Of these, five trials evaluated the continuation of ACEi/ARB therapies in those already on such therapies compared with discontinuation, and 14 trials involved initiation of ACEi/ARB therapies in those not on such therapies. Eleven trials did not meet inclusion criteria due to (1) not assessing ACEi/ARB therapies $(n=6)$, (2) absence of a control group ( $n=2)$, (3) use of non-oral administration of ACEi/ ARB therapies $(n=2)$ or (4) recruitment of patients with active malignancy $(n=1)$. We extracted information about eligible investigators to invite them to participate in the prospective meta-analysis by contributing tabular data. An invitation letter will be sent via email and will include details about the study protocol, offer of authorship, identification of the time for data retrieval and confirmation of data security.

This search will be continuously updated, and investigators of newly reported and eligible trials will be invited to join the collaboration. Using the Cochrane Collaboration guidelines, ${ }^{25}$ electronic searches of MEDLINE (1996present), EMBASE (1996-present) and the Cochrane Central Register of Controlled Trials (most recent edition) will also be performed to identify any other RCTs that meet inclusion criteria in March 2021. A comprehensive search strategy using MeSH terms and text words will be used (online supplemental table 1). Two investigators (SRG and AES) will screen the abstracts for potential inclusion against the eligibility criteria, and screen the full texts. Reference management software (EndNote) will be used to store identified records.

All provided data will be stored on a password protected server, with limited access only to those directly involved in data analysis.

\section{Study evaluation}

A quality assessment of each RCT will be performed independently by two authors (SRG and AES), with disagreements resolved by discussion. This will include evaluation of allocation sequence, allocation concealment, blinding, lost to follow-up and completeness of outcome reporting. ${ }^{25}$ Randomisation procedures, treatment allocation according to assignment, outcomes collected and compared across groups; blinding methods; and risk of bias both at study and outcome levels will also be assessed using the Cochrane Risk of Bias Tool. ${ }^{26}$ Two investigators (SRG and AES) will independently review the articles and any disagreements will be adjudicated by a third independent investigator (AR).

\section{Statistical analysis}

Trial-specific outcome data will be pooled. For binary outcomes, risk ratios and 95\% CI will be estimated using log-binomial mixed-effects models (or ORs from logistic models as required). Key results will be presented using Forest plots, and the $\mathrm{I}^{2}$ statistic will be used to quantify the degree of heterogeneity between studies. ${ }^{27} \mathrm{~A}$ two-tailed $\mathrm{p}$ value of $5 \%$ will be used for hypothesis testing. A fixed effects analysis will be used unless there is significant 


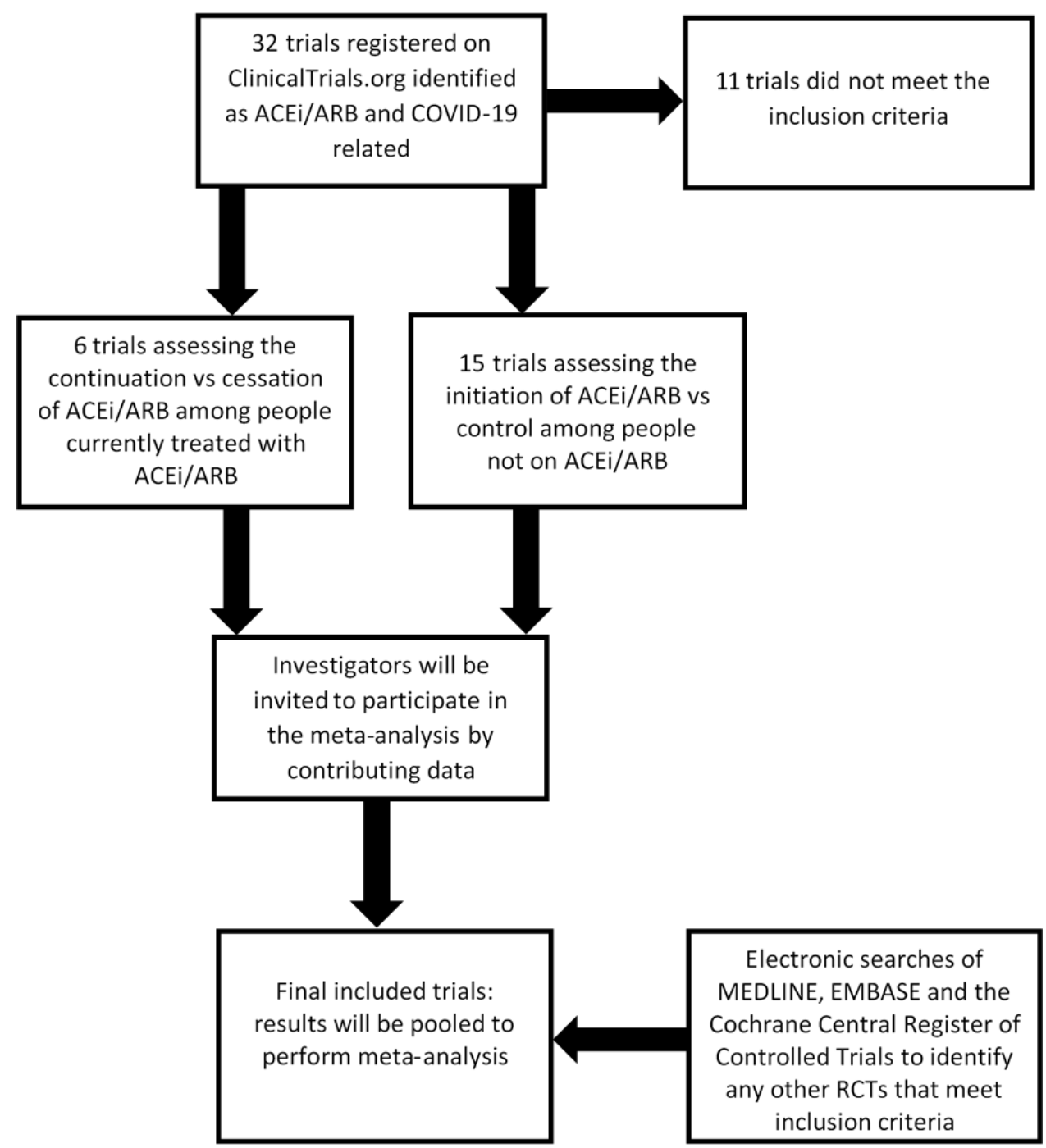

Figure 1 Flow chart of trial inclusion. ACEi, ACE inhibitors; ARB, angiotensin II receptor blocker; RCTs, randomised controlled trials.

heterogeneity (as evidenced by $\mathrm{I}^{2}>70 \%$ and quantitatively large variation), in which case pooling will not be performed. ${ }^{28}$ If heterogeneity is found, we will attempt to determine potential reasons by examining characteristics of individual trials. Potential outliers will be investigated in a sensitivity analysis by dropping each study at a time. Publication bias will be assessed by visual inspection of funnel plots and formally using Egger's method to evaluate the tendency of publishing studies with statistically significant findings. ${ }^{29}$ We will use meta-regression analyses to further explore heterogeneity of treatment effects if considerable residual heterogeneity remains after controlling for variables. Analyses will also be stratified by the prespecified subgroup analysis as mentioned below. In general, reporting of the findings will follow PRISMA guidelines. ${ }^{24}$ All analyses will be conducted using Review Manager V.5.3 software (Copenhagen, The Nordic Cochrane Centre, The Cochrane Collaboration, 2014).

There is potential heterogeneity in trial inclusion criteria, which may affect the meta-analysis findings and be a source of bias. Therefore, subgroup analyses will be performed depending on data availability to explore differences in effects by partitioning by study-level categorical covariates. We will aim to assess all-cause mortality at $\leq 30$ days and $>1$ month based on the following stratifications: (1) sex: male versus female; (2) age: $<60$ years versus $\geq 60$ years; (3) comorbidities: hypertension vs no hypertension, diabetes versus no diabetes, CKD defined as eGFR $<60 \mathrm{~mL} / \mathrm{min} / 1.73 \mathrm{~m}^{2}$ versus no $\mathrm{CKD}$, cardiovascular disease (CVD) (defined as established coronary artery disease, heart failure, arrythmia and/ or stroke) versus no CVD, chronic obstructive pulmonary disease versus no chronic obstructive pulmonary disease; (4) smoking status: ever smoked versus nonsmokers; (5) hospitalisation status: hospitalised versus non-hospitalised patients; (6) ethnicity: white, SouthEast/East Asian, South Asian, African and Other; (7) trial type: randomised trials that investigate continuation and cessation of ACEi/ARBs among patients currently treated with ACEi/ARBs, versus trials that investigate initiation of ACEi/ARBs in those not currently treated with such therapies compared with control; (8) region: 
Table 2 Potential eligible randomised controlled trials of adults with COVID-19

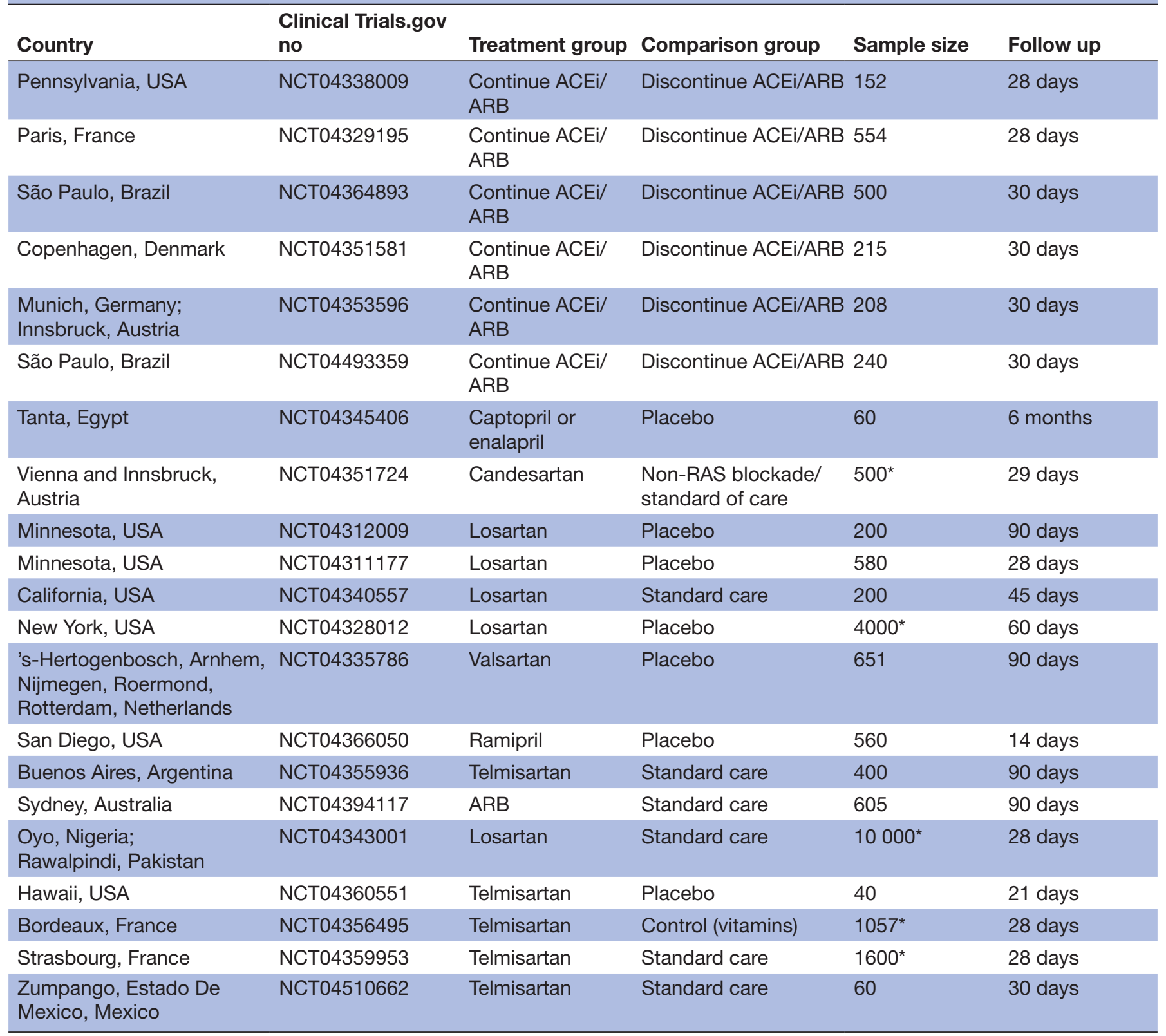

*Sample size consists of multiple trial arms including non-ACEi/ARB therapies or non-control groups.

ACEi, ACE inhibitors; ARB, angiotensin II receptor blockers; RAS, renin-angiotensin system.

Americas, Europe, Africa, South and West Asia/Middle East, and North Asia/South-East Asia/Oceania; (9) ACEi and ARB separately if feasible as pooling ACEi and ARB together does not account for between-class differences; (10) follow-up period: final short-term follow-up 14 days compared with final follow-up at 28-30 days and (11) asymptomatic versus symptomatic clinical status (online supplemental tables 2 and 3 ).

\section{Data statement}

We will not have access to identifiable patient information. All data sharing and storage procedures will be compliant with the Australian Data Privacy Act of 1988. All trial data will be considered confidential and will not be provided to any third party. Data will be stored on site at The George Institute for Global Health, King St Campus, Sydney, Australia, with strict confidentiality and comprehensive data security.

\section{Limitations}

Limitations of our meta-analysis reflect the limitations of the included RCTs, and are outside the control of our planned analyses. We have attempted to be inclusive as possible and aim to perform subgroup analyses given the trials are mostly small and may not be powered for such analyses. Key limitations include the relatively short follow-up duration of included trials (most $\leq 30$ days), which prevent robust assessment of long-term outcomes 
such as cardiovascular events and overall mortality. Additionally, the majority of patients on ACEi/ARBs will likely be on such therapy to treat hypertension (although this has not been specified in the inclusion trials of the eligible RCTs) and the generalisability of our findings to cardiac failure and renal disease may be uncertain if there are insufficient numbers.

\section{Ethics and dissemination}

All individual trials will require Ethics Committee or Institution Review Board approval. We will publish our findings in peer-reviewed medical journals. Publications will be in the name of the collaborative group involving all trialists who provided data. Further, we will share and present our findings at scientific meetings and through the networks and memberships across professional societies.

\section{Implications of the review}

Given the widespread use of ACEi and ARBs worldwide, guidance on the use of ACEi/ARBs in adults with COVID-19 is urgently needed, relying on evidence beyond observational data. This collaborative international approach will allow dissemination of results, which will inform public health policy and clinical decision making for ongoing ACEi/ARBs use on an international scale. Healthcare providers and policy-makers can use the findings to improve the clinical decision making by developing strategies and guidelines to guide use of ACEi/ ARBs in hypertension, CVD and kidney disease.

\section{Patient and public involvement}

Patients were not involved in the development of the research question, outcome measure and study design.

\section{Author affiliations}

${ }^{1}$ The George Institute for Global Health; University of New South Wales, Sydney, New South Wales, Australia

${ }^{2}$ Department of Medical and Surgical Sciences, University of Bologna, Bologna, Italy ${ }^{3}$ Department of Cellular and Molecular Medicine, Kidney Research Centre, Ottawa Hospital Research Institute, University of Ottawa, Ottawa, Ontario, Canada ${ }^{4}$ School of Health and Life Sciences, Federation University Australia, Ballarat, Victoria, Australia

${ }^{5}$ Imperial Clinical Trials Unit, Imperial College London, London, UK

${ }^{6}$ Dobney Hypertension Centre, School of Medicine, Royal Perth Hospital Unit, University of Western Australia, Perth, Western Australia, Australia

${ }^{7}$ Department of Cardiovascular and Renal Research, University of Southern Denmark, Odense, Denmark

${ }^{8}$ Third Department of Medicine, Hypertension Center STRIDE-7, School of Medicine, Sotiria Hospital, National and Kapodistrian University of Athens, Athens, Greece ${ }^{9}$ Division of Medicine and Manchester Academic Health Science Centre, Manchester University NHS Foundation Trust Manchester, Manchester, UK

${ }^{10}$ CARIM-School for Cardiovascular Diseases, Maastricht University, Maastricht, The Netherlands

${ }^{11}$ Department of Pharmacology and Experimental Therapeutics and the Whitaker Cardiovascular Institute, Boston University School of Medicine, Boston, Massachusetts, USA

${ }^{12}$ Institute of Cardiovascular Science, University College London and National Institute for Health Research (NIHR) University College London Hospitals Biomedical Research Centre, London, UK

Twitter Aletta E Schutte @alta_schutte

Acknowledgements Stella Galanis, NSW Health librarian.
Contributors SRG, AR and AES were involved in the planning and writing of this protocol. CB, DB, FC, NRP, MPS, UMS, GS, MT, TU, RDW and BW reviewed the protocol and provided intellectual input.

Funding The authors have not declared a specific grant for this research from any funding agency in the public, commercial or not-for-profit sectors.

Competing interests None declared.

Patient consent for publication Not required.

Provenance and peer review Not commissioned; externally peer reviewed.

Supplemental material This content has been supplied by the author(s). It has not been vetted by BMJ Publishing Group Limited (BMJ) and may not have been peer-reviewed. Any opinions or recommendations discussed are solely those of the author(s) and are not endorsed by BMJ. BMJ disclaims all liability and responsibility arising from any reliance placed on the content. Where the content includes any translated material, BMJ does not warrant the accuracy and reliability of the translations (including but not limited to local regulations, clinical guidelines, terminology, drug names and drug dosages), and is not responsible for any error and/or omissions arising from translation and adaptation or otherwise.

Open access This is an open access article distributed in accordance with the Creative Commons Attribution Non Commercial (CC BY-NC 4.0) license, which permits others to distribute, remix, adapt, build upon this work non-commercially, and license their derivative works on different terms, provided the original work is properly cited, appropriate credit is given, any changes made indicated, and the use is non-commercial. See: http://creativecommons.org/licenses/by-nc/4.0/.

\section{ORCID iDs}

Sonali Rukshana Gnanenthiran http://orcid.org/0000-0003-4145-6461

Bryan Williams http://orcid.org/0000-0002-8094-1841

Aletta E Schutte http://orcid.org/0000-0001-9217-4937

\section{REFERENCES}

1 Gu Q, Burt VL, Dillon CF, et al. Trends in antihypertensive medication use and blood pressure control among United States adults with hypertension: the National health and nutrition examination survey, 2001 to 2010. Circulation 2012;126:2105-14.

2 Li W, Moore MJ, Vasilieva N, et al. Angiotensin-converting enzyme 2 is a functional receptor for the SARS coronavirus. Nature 2003;426:450-4.

3 Hoffmann M, Kleine-Weber H, Schroeder S, et al. SARS-CoV-2 cell entry depends on ACE2 and TMPRSS2 and is blocked by a clinically proven protease inhibitor. Cell 2020;181:271-80.

4 Ferrario CM, Jessup J, Chappell MC, et al. Effect of angiotensinconverting enzyme inhibition and angiotensin II receptor blockers on cardiac angiotensin-converting enzyme 2. Circulation 2005;111:2605-10.

5 Igase M, Kohara K, Nagai T, et al. Increased expression of angiotensin converting enzyme 2 in conjunction with reduction of neointima by angiotensin II type 1 receptor blockade. Hypertens Res 2008;31:553-9.

6 Ocaranza MP, Moya J, Barrientos V, et al. Angiotensin-(1-9) reverses experimental hypertension and cardiovascular damage by inhibition of the angiotensin converting enzyme/Ang II axis. J Hypertens 2014;32:771-83.

7 Fang L, Karakiulakis G, Roth M. Are patients with hypertension and diabetes mellitus at increased risk for COVID-19 infection? Lancet Respir Med 2020;8:e21.

8 Sommerstein R, Grani C. Preventing a COVID-19 pandemic: ACE inhibitors as a potential risk factor for fatal COVID-19. BMJ 2020;368:m810.

9 Sparks MA, South A, Welling P, et al. Sound science before quick judgement regarding Ras blockade in COVID-19. Clin J Am Soc Nephrol 2020;15:714-6.

10 de Abajo FJ, Rodríguez-Martín S, Lerma V, et al. Use of reninangiotensin-aldosterone system inhibitors and risk of COVID-19 requiring admission to hospital: a case-population study. Lancet 2020;395:1705-14

11 Jung S-Y, Choi JC, You S-H, et al. Association of Renin-angiotensinaldosterone System Inhibitors With Coronavirus Disease 2019 (COVID-19)- Related Outcomes in Korea: A Nationwide Populationbased Cohort Study. Clin Infect Dis 2020;71:2121-2128.

$12 \mathrm{Li} \mathrm{J}$, Wang X, Chen J, et al. Association of renin-angiotensin system inhibitors with severity or risk of death in patients with hypertension hospitalized for coronavirus disease 2019 (COVID-19) infection in Wuhan, China. JAMA Cardiol 2020;5:825-30. 
13 Mancia G, Rea F, Ludergnani M, et al. Renin-angiotensin-Aldosterone system blockers and the risk of COVID-19. N Engl J Med 2020;382:2431-40.

14 Reynolds HR, Adhikari S, Pulgarin C, et al. Renin-angiotensinAldosterone system inhibitors and risk of COVID-19. N Engl J Med 2020;382:2441-8.

15 Pirola CJ, Sookoian S. Estimation of renin-angiotensin-aldosteronesystem (RAAS)-inhibitor effect on COVID-19 outcome: a metaanalysis. J Infect 2020;81:276-81.

16 Guo X, Zhu Y, Hong Y. Decreased mortality of COVID-19 with reninangiotensin-aldosterone system inhibitors therapy in patients with hypertension: a meta-analysis. Hypertension 2020;76:e13-14.

17 Zhang P, Zhu L, Cai J, et al. Association of inpatient use of angiotensin-converting enzyme inhibitors and angiotensin II receptor blockers with mortality among patients with hypertension hospitalized with COVID-19. Circ Res 2020;126:1671-81.

18 Felice C, Nardin C, Di Tanna GL, et al. Use of RAAS inhibitors and risk of clinical deterioration in COVID-19: results from an Italian cohort of 133 hypertensives. Am J Hypertens 2020;33:944-8.

19 Internation Society of Hypertension. Statement on COVID-19, 2020. Available: https://ish-world.com/news/a/A-statement-from-theInternational-Society-of-Hypertension-on-COVID-19

20 American College of Cardiology, American Heart Association and Heart Failure Society of America. Patients taking ACE-i and Arbs who contract COVID-19 should continue treatment, unless otherwise advised by their physician, 2020. Available: https://www.acc.org/ latest-in-cardiology/articles/2020/03/17/08/59/hfsa-acc-ahastatement-addresses-concerns-re-using-raas-antagonists-in-covid19

21 European Society of Cardiology Council on Hypertension. Position statement of the ESC Council on hypertension on ACE-inhibitors and angiotensin receptor blockers, 2020. Available: https://www. escardio.org/Councils/Council-on-Hypertension-(CHT)/News/ position-statement-of-the-esc-council-on-hypertension-on-aceinhibitors-and-ang

22 European Society of Hypertension. Statement on COVID-19, 2020. Available: https://www.eshonline.org/spotlights/esh-stabtement-oncovid-19

23 The Renal Association. UK position statement on COVID-19 and ACE Inhibitor/Angiotensin receptor blocker use, 2020, 2020. Available: https://renal.org/covid-19/ra-resources-renalprofessionals/renal-association-uk-position-statement-covid-19-aceinhibitorangiotensin-receptor-blocker-use

24 Liberati A, Altman DG, Tetzlaff J, et al. The PRISMA statement for reporting systematic reviews and meta-analyses of studies that evaluate healthcare interventions: explanation and elaboration. BMJ 2009;339:b2700.

25 Higgins J, Thomas J, Chandler J, et al, eds. Cochrane Handbook for systematic reviews of interventions version 6.0 (updated July 2019). London: Cochrane, 2019.

26 Higgins JP, Altman DG, Gotzche P. Cochrane bias methods group; cochrane statistical methods group. The cochrane collaboration's tool for assessing risk of bias in randomised trials. $B M J$ 2011;343:1-9.

27 Higgins JPT, Thompson SG. Quantifying heterogeneity in a metaanalysis. Stat Med 2002;21:1539-58.

28 Hedges LV, Vevea JL. Fixed- and random-effects models in metaanalysis. Psychol Methods 1998;3:486-504.

29 Egger M, Davey Smith G, Schneider M, et al. Bias in meta-analysis detected by a simple, graphical test. BMJ 1997;315:629-34. 